\title{
A multi-timescale hybrid stochastic/deterministic generation scheduling framework with flexiramp and cycliramp costs
}

\author{
Mohamed Shaaban ${ }^{\mathrm{a}, \mathrm{b}, *}$, Wen-Shan Tan ${ }^{\mathrm{c}}$, Md. Pauzi Abdullah ${ }^{\mathrm{c}}$ \\ a Department of Electrical \& Electronic Engineering, Faculty of Engineering, Universiti Malaysia Sarawak, 94300 Kota Samarahan, Sarawak, Malaysia \\ ${ }^{\mathrm{b}}$ Department of Electrical Engineering, Faculty of Engineering, Port Said University, Port Fouad 42526, Port Said, Egypt \\ ${ }^{\mathrm{c}}$ Centre of Electrical Energy Systems, Institute of Future Energy, Universiti Teknologi Malaysia, 81310 Johor Bahru, Malaysia
}

\section{A R T I C L E I N F O}

\section{Keywords:}

Flexible ramping product

Generation unit ramping cost

Ramp market

Renewable energy integration

Mixed-integer linear programming

\begin{abstract}
A B S T R A C T
Flexible ramping products (flexiramp), provided by entitled resources to meet net demand forecast error, are the underpinning for the accommodation of the substantial uncertainties associated with variable wind power. This paper proposes an enhanced flexiramp modeling approach, cast in a hybrid stochastic/deterministic multitimescale framework. The framework employs a chance-constrained day-ahead scheduling method, as well as deterministic scheduling on intra-hourly basis (real-time scheduling), to allow optimal procurement planning of the flexiramp products in both timescales. A stepwise and piecewise demand price curve is also proposed to calculate the flexiramp surplus procurement price. Non-generation resource (NGR), referring to energy storage, is implemented to provide extra flexibility. Additionally, cycling ramping cost (cycliramp), introduced to model operational and maintenance costs and reduce the wear and tear of generators, is also included as a penalty. Numerical tests are conducted on 6-bus and 118-bus systems. Results demonstrate the merits of the proposed scheduling model as well as the effects of flexiramp and cycliramp costs in the multi-timescale scheduling.
\end{abstract}

\section{Introduction}

Flexibility issues are drawing increased attention due to the growing penetration levels of variable renewable generation. Such a challenge is more imminent with the variability and uncertainty of wind generation, especially in the intra-hour timescales, which may lead to difficulties in energy balancing, and a compromise on power system's efficiency and reliability [1]. Variability is defined in this paper as the difference of expected net load between time intervals, while uncertainty is the unpredictability or the net load forecast error.

Along with the requirement to improve power system flexibility, a new market product, called the flexible ramping (flexiramp), has been recently proposed by CAISO [2,3] and MISO [4] to accommodate net load uncertainty. Currently, CAISO only procures flexiramp in the deterministic short-term scheduling, while MISO procures it in both deterministic day-ahead and real-time scheduling. Flexiramp, also known as "ramp capacity" (as in MISO), is defined as the sufficient ramping capacity provided by eligible resources in time interval $t$ to meet the upward and downward net load forecast error in the subsequent intervals, $t+1$, with a high confidence level [1-4].

Cycling, defined as the changes in the power output of conventional thermal units (ramping or on/off switching), is the source of operational flexibility in the electricity generation system [5]. Along with the introduction of flexiramp market and the increase in net load variability, frequent cycling ramp (cycliramp) would result in additional planned outages and higher operation and maintenance (O\&M) costs [6]. Cycliramp cost, as an inherent feature of thermal units, ought to be considered explicitly in flexiramp markets.

Multi-timescale scheduling is becoming a regular practice in power system markets [7]. It mainly consists of; (a) day-ahead scheduling, which runs every $24 \mathrm{~h}$ at 1 -h time resolution. (b) real-time or hourlyahead online rolling scheduling that is performed every $1 \mathrm{~h}$, to determine the generation output in the upcoming 3 or $4 \mathrm{~h}$, with a time resolution of 15-min. Real-time scheduling can use the newly updated information of load profile, weather forecast and wind power generation to improve the prediction precision.

\subsection{Flexible ramp capacity market}

Research on flexible ramping products started only recently, focusing on generation scheduling and economic dispatch, with deterministic models $[8,9]$ and stochastic models $[8,10,11]$. Wang and Hobbs [10] conducted a comparative analysis for a deterministic flexiramp dispatch model versus a stochastic model. Results demonstrated

\footnotetext{
* Corresponding author at: Department of Electrical \& Electronic Engineering, Faculty of Engineering, Universiti Malaysia Sarawak, 94300 Kota Samarahan, Sarawak, Malaysia.

E-mail address: mshaaban@unimas.my (M. Shaaban).
} 


\author{
Nomenclature \\ Parameters \\ $p_{i}^{\max (\min )} \max (\min )$ generation of unit $i[\mathrm{MW}]$ \\ $\phi^{u p(d n)} \quad$ up(down) flexiramp surplus procurement [MW] \\ $c_{f}^{\text {frus(frds) }}$ price of step $f$ of up(down) flexiramp surplus award \\ [\$/MWh] \\ $\chi_{f}^{\max , u p(d n)}$ step size $f$ of up(down) flexiramp surplus [MW] \\ $d_{s, t}^{\text {net }} \quad$ net load discrete realization level $s$ at time $t$ [MW] \\ $\varepsilon^{u p(d n)} \quad$ up(down) flexiramp surplus confidence level \\ $h r_{i}^{g} \quad$ incremental heat rate of unit $i[\mathrm{Btu} / \mathrm{kWh}]$ \\ $\pi_{i}^{g} \quad$ incremental fuel cost of unit $i$ [\$/Btu] \\ $s r_{t} \quad$ spinning reserve at time $t$ [MW] \\ $T_{i}^{\text {on(off) }} \quad$ minimum on/off time of unit $i$ [hour] \\ $q_{j}^{\max , c(d)} \quad$ max charge(discharge) rate of storage $j$ [MWh] \\ $q_{j}^{\min , c(d)} \quad$ min charge(discharge) rate of storage $j$ [MWh] \\ $e_{j, m}^{\max (\min )} \max (\min )$ capacity of storage $j$ at time $m$ [MWh] \\ $E_{j}^{0} \quad$ initial state of charge (SOC) of storage $j$ at the initial \\ horizon [\%] \\ $\eta_{j}^{c(d)} \quad$ efficiency rate to charge/(discharge) of storage $j$ \\ $\tau \quad$ real-time slot $[\mathrm{h}]$ \\ $p_{m}^{w, \max } \quad$ maximum wind generation at time $m$ [MW] \\ Binary variables at time $t$ /time $m$

$\begin{array}{ll}o_{i, t(m)} & \text { on/off status of units } i \\ x_{j, t(m)} & \text { charge/discharge status of storage } j \\ \gamma_{s, t} & \text { expected net load auxiliary variable of level } s \\ \alpha_{s, t}, \beta_{s, t} & \text { ramp up, down auxiliary variable of level } s \\ z_{s, t} & \text { probabilistic auxiliary variable of level } s \\ y_{h, t} & \text { probabilistic auxiliary variable of level } h\end{array}$

Continuous variables at time $t /$ parameters at time $m$

$r_{i, t(m)}^{u p(d n)} \quad$ ramp up(down) rate limit of unit $i$ [MWh] $p_{i, t(m)}^{(D A, s c h)}$

[MW]

$q_{j, t}^{c(d)} \quad$ charge(discharge) rate of storage $j$ [MWh]

$e_{j, t(m)}^{(D A, s c h)} \quad$ (Day-ahead, online rolling schedule) SOC of storage $j$ [\%]

$\bar{d}_{t}^{\text {net }} \quad$ expected net load [MW]

$F R U_{i(j), t}$ up flexiramp award of unit $i$ (storage $j$ ) [MW]

$F R D_{i(j), t}$ down flexiramp award of unit $i$ (storage $j$ ) [MW]

$C R U_{i, t} \quad$ up cycliramp award of unit $i$ [MW]

$C R D_{i, t} \quad$ down cycliramp award of unit $i$ [MW]

$F R U S_{t(m)}^{(D A, s c h)}$ (Day-ahead, online rolling schedule) up flexibility surplus award [MW]

$F R D S_{t(m)}^{(D A, s c h)}$ (Day-ahead, online rolling schedule) down flexibility surplus award [MW]

$F R U R_{t} \quad$ up flexibility reserve award [MW]

$F R D R_{t} \quad$ down flexibility reserve award [MW]

$\omega_{s, t} \quad$ linearization variable of level $s$

$g_{j, t} \quad$ PDR auxiliary variable

$p r_{s, t} \quad$ probability of net load level $s$

\section{Continuous variables at time $m$}

$\Delta_{i, m}^{p g} \quad$ generation deviation of unit $i[\mathrm{MW}]$

$\Delta_{i, m}^{e} \quad$ SOC deviation of storage $j[\%]$

$\Delta_{m}^{\text {frus (frds) }}$ up/down flexiramp surplus deviation [MWh]

$\Delta_{i}^{\text {max } p g} \quad$ deviation limit of generation unit $i$ in [MW]

$\Delta_{j}^{\text {max }, e} \quad$ deviation limit of SOC of storage $j$ [\%]

$\Delta_{j}^{\text {max.frus(frds) }}$ deviation limit of up(down) flexiramp surplus [MWh]

Matrices and vectors

SF shift factor

$P L^{\max } \quad$ vector of upper limit for power flow

$P^{G}, P^{D} \quad$ vector of generation dispatch, load demand

$K_{G}, K_{D}$ bus-generator, bus-load incident matrix that the deterministic model is inefficient and the amount of flexiramp procurement strongly affects results. Marneris et al. have proposed deterministic and stochastic scheduling models in [8], considering the variability and uncertainty reserves, in a day-ahead scheduling model with 60 and $15 \mathrm{~min}$ intra-hour timescales. The proposed model of intrahour uncertainty and variability reserves could be massive, since they were predicted day-ahead. The respective reserves were not re-allocated in a real-time dispatch model, however, which may lead to inefficient flexiramp procurement. In addition, the day-ahead model utilizes full stochastic programming without transmission network constraints. Stochastic programming suffers from the dimensionality problem, leading to a long computation time, even with the nine net load scenarios considered. Wu et al. [11] incorporated flexiramp costs in a security-constrained stochastic scheduling along with other nongeneration resource (NGR) options, such as energy storage (ES), in providing flexiramp. However, the proposed flexiramp cost function model is rather generic, since the formulation considers cycliramp and flexiramp surplus as an aggregate amount, to cope with the net load variability and uncertainty.

\subsection{Cycling ramp cost}

The work presented herein also fits into an uptrend in the literature on generation cycling cost $[5,6,12,13]$. Increased cycliramp with rising wind penetration levels, as established in [12], causes growing concern about wear-and-tear of thermal generation and the related O\&M costs. Current market operation practices consider thermal generation ramp rate constraints rather than cycliramp cost. The additional cost sustained by generation companies (GENCOs) for frequent ramping to compensate for the net load variability, are not included with the ramp rate constraints [13]. Troy et al. [14] presented linear, piecewise and step-shaped long-term cycling start-up and cycliramp cost functions. Results showed an overall saving for the system as the cycling operation was subsequently reduced. On the other hand, generation commitment may be altered as new generator units will have a much lower cycling cost. Wu et al. [6] proposed an energy based cycliramp calculation with demand response in a day-ahead stochastic scheduling model. The resultant model is nonlinear and was solved with the MIQCP solver. The latter is not in tandem with state-of-the-art MILP models currently adopted by most ISOs $[2,4]$.

\subsection{Aims and contributions of this paper}

Although the literature is not lacking in the modeling and application analysis regarding the implementation of flexiramp product in realtime market [10], a committed study of the respective product procurement planning in a hybrid day-ahead stochastic model and realtime deterministic model, is not adequately addressed yet. Two important issues need to be specifically addressed in such undertaking: (a) the necessity for an optimal positioning of the day-ahead flexiramp procurement, in order to efficiently coordinate and respond to net load deviation and the uncertainty in real-time operation. (b) the need for appropriate penalties or demand curves that would ensure optimal flexiramp to be acquired and avoid over-procurement. 
This paper proposes a hybrid stochastic/deterministic multi-timescale scheduling (HSD-MTS) model, which consists of day-ahead stochastic scheduling optimization (DASO) and real-time deterministic scheduling optimization (RTDO), for flexiramp procurement with cycliramp constraints. In contrast to [8], the proposed DASO approach employs chance-constrained programming (CCP) to maintain the computational tractability, along with an efficient flexiramp procurement model. Furthermore, the two important issues singled out previously were fully addressed in this paper. The contribution of the paper can be summarized as follows:

(a) A new flexiramp reserve concept is introduced for the multi-timescale scheduling, to avoid over procurement of flexiramp surplus, while making sure the system flexiramp capacity is sufficient to deal with any scenario in real-time scheduling.

(b) In compliance with the CAISO flexiramp market planning proposition [2], a flexiramp surplus piecewise cost function (demand curve) is constructed, with real-time net demand forecast error, and implemented in both day-ahead flexiramp planning and real-time flexiramp surplus procurement. The flexiramp surplus cost function tends to minimize the aftereffect of wind curtailment; which is modeled as a decision variable to provide extra degrees of freedom to meet the constraints.

(c) An enhanced flexiramp quantification method is developed, by separating the net load uncertainty into up and down flexiramp regions, for the DASO CCP model. The proposed method ensures the accuracy and elasticity of flexiramp procurement, to align the dayahead flexiramp amount with the real-time net load uncertainty.

(d) Energy storage (ES), representing non-generation resources, is formulated as a flexiramp product. The balance between the flexiramp and cycliramp in the generation scheduling with the ES is further investigated.

The paper is organized as follows. Flexiramp and cycliramp modeling, along with the flexiramp price curve is discussed in Section 2, Section 3 presents the DASO CCP formulation, whereas the RTDO formulation is presented in Section 4. Case studies and numerical results are given in Section 5. Section 6 concludes.

\section{Flexiramp and cycliramp modelling}

\subsection{Flexiramp surplus and cycliramp}

Power system operation requires more flexible resources to balance the gradually rising variable generation. The combination of uncertainties from both load and variable generation, referred to as net load forecast error, leads to the need for flexiramp capability, especially in the real-time horizon to ensure system reliability. Fig. 1 depicts a typical day-ahead scheduling of thermal generating units, which are scheduled to ramp up across time $t$ to $t+4$, in a 15-min timescale. For instance, the day-ahead flexiramp procurements (blue dotted lines) in Fig. 1, are set to a preset value $\phi$, which is set to $95 \%$ confidence level of the real-time forecast error (red dotted lines), whereas total flexiramp capacity requirement is set to $75 \%$ confidence level (blue dashed lines). The sources of net load variability come from the expected net load movement across time intervals, mainly for load following. Therefore, cycliramp (CRU/CRD) is referred to the ramping requirement due to the load following in a time period. Instead, flexiramp surplus (FRUS/FRDS) is defined as the generation ramping that can be obtained to meet the net load uncertainty, within a specified level in a certain period of time. Flexiramp reserve (FRUR/FRDR) is the remaining flexiramp from the total flexiramp requirement within a confidence range. It is kept to meet the smaller timescale net load flexiramp requirement in various scenarios.

The generation flexiramp capacity available in a time period is expressed as:

$$
\begin{aligned}
& F R U_{i, t}=\min \left(r_{i, t}^{u p}, o_{i, t} \cdot\left(p_{i}^{\max }-p_{i, t}\right)\right) \\
& F R D_{i, t}=\min \left(r_{i, t}^{d n}, o_{i, t} \cdot\left(p_{i, t}-p_{i}^{\min }\right)\right)
\end{aligned}
$$

The NGR unit, ES, is included in this paper to provide extra flexiramp capability, which can be expressed as:

$F R U_{j, t}=\min \left(q_{j, t}^{\max , d},\left(e_{j, t} \cdot e_{j}^{\max }-e_{j}^{\min }\right) \cdot \eta_{j}^{d}\right)$

$F R D_{j, t}=\min \left(q_{j, t}^{\max , c},\left(1-e_{j, t}\right) \cdot e_{j}^{\max } \cdot\left(1 / \eta_{j}^{c}\right)\right)$

Eqs. (3) and (4) denote that the ES upward/downward flexiramp capacity are equal to the ES discharge/charge rate respectively, and is limited by the remaining SOC level. The charge/discharge efficiencies are also taken into consideration. A low efficiency ES denotes that $1 \%$ of the SOC level will produce a lower amount of energy to the grid (lower upward flexiramp) and requires higher energy from the grid to charge the ES (higher downward flexiramp).

The up and down cycliramp of a generation unit is obtained excluding generation start-up or shut-down costs, since the cycling cost for those actions are already included in the generation start-up and shut-down cost function.

$C R U_{i, t}=\max \left(0, p_{i, t+1}-p_{i, t}-\left|\left(o_{i, t+1}-o_{i, t}\right) \cdot p_{i}^{\max }\right|\right)$

$C R D_{i, t}=\max \left(0, p_{i, t}-p_{i, t+1}-\left|\left(o_{i, t+1}-o_{i, t}\right) \cdot p_{i}^{\max }\right|\right)$

Summation of the flexiramp surplus and cycliramp in time $t$, is set to be bounded by the minimum and maximum limits. The former is the flexiramp surplus procurement preset value $\phi(95 \%$ of the real-time forecast error), whereas the latter represents total system flexiramp capacity from multiple sources, expressed as:

$\phi^{u p} \leqslant F R U S_{t}+\sum_{i=1}^{I}\left[C R U_{i, t}\right] \leqslant \sum_{i, j=1}^{I, J}\left[F R U_{i, t}+F R U_{j, t}\right]$

$\phi^{d n} \leqslant F R D S_{t}+\sum_{i=1}^{I}\left[C R D_{i, t}\right] \leqslant \sum_{i, j=1}^{I, J}\left[F R D_{i, t}+F R D_{j, t}\right]$

$F R U R_{t}=\sum_{i, j=1}^{I, J}\left[F R U_{i, t}+F R U_{j, t}\right]-F R U S_{t}-\sum_{i=1}^{I}\left[C R U_{i, t}\right]$

$F R D R_{t}=\sum_{i, j=1}^{I, J}\left[F R D_{i, t}+F R D_{j, t}\right]-F R D S_{t}-\sum_{i=1}^{I}\left[C R D_{i, t}\right]$

Flexiramp reserve, in (9) and (10), is needed to ensure the system has enough flexibility to provide for the smaller timescale flexiramp requirement (i.e., RTDO), due to changes in net load movement. The preset value of flexiramp surplus, $\phi$, is set to $95 \%$ of the real-time

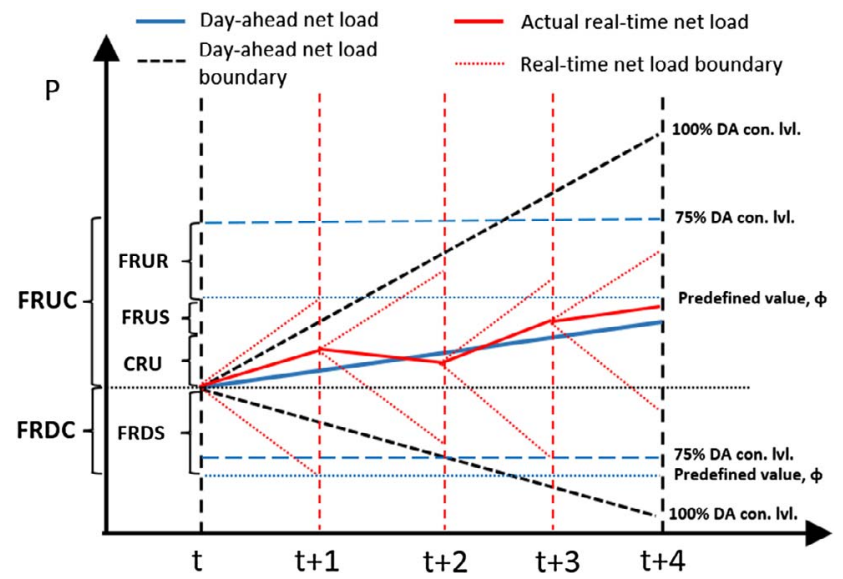

Fig. 1. Day-ahead upward ramp in hybrid stochastic/deterministic multi-timescale model. 
forecast error uncertainty of time $t+1$ (first 15-min interval for each hour), as in Fig. 1. It can be set to a higher level, albeit an over procurement, in other time periods, maybe triggered causing additional opportunity cost. The preset value $\phi$ will make sure the FRUS, procured in DASO, is close to the real-time FRUS requirement. This will be beneficial to the HSD-MTS simulation, as will be explained in Section 4. In case the preset value $\phi$ is larger than the day-ahead chance constraint confidence level, as in the FRDS in Fig. 1, the preset value $\phi$ will be procured as flexiramp surplus and there is no flexiramp reserve available.

Higher flexiramp procurement will incur additional cost. Therefore, in order to preserve the cost effectiveness of the flexiramp product, in this paper, the proposed model procures flexiramp capacity partially with $75 \%$ of confidence level in DASO. The preset value of flexiramp surplus $\phi$, along with the proposed flexiramp reserve, will ensure efficient procurement, and avoid over or under procurement of flexiramp surplus in the day-ahead scheduling model.

In summary, the total flexiramp capacity available to accommodate wind uncertainty in the day-ahead is equal to the summation of flexiramp surplus and flexiramp reserve (limited by the preset value $\phi$ and the chance constraint confidence level respectively). On the other hand, the total flexiramp capacity in the real-time is always equal to $95 \%$ of the real-time forecast error.

\subsection{Flexiramp demand price curve}

In compliance with [2], for a $800 \mathrm{MW}$ wind farm, a flexiramp demand price curve is uniquely proposed in this paper. The curve is approximated by a net load error histogram constructed from the Autoregressive Moving Average (ARMA) forecasted real-time net load, as shown in Table 1 . The piecewise curve, denoted by a solid line, reflects the flexiramp relationship directly against the procurement cost.

Assuming the maximum net load forecast error is $400 \mathrm{MW}$, without a flexiramp surplus cost function, the probability of upward flexiramp requirement falling within $0 \mathrm{MW}$ to $100 \mathrm{MW}$ is 0.45 . Conversely, the flexiramp surplus procured is between $300 \mathrm{MW}$ and $400 \mathrm{MW}$, which leads to the $C_{\text {frus }}=\operatorname{pr}(F R U S) *$ penalty cost, where the penalty cost is the maximum energy price for regulation service procurement, $\$ 1000$ / MWh and \$150/MWh for up and down flexiramp respectively. This warrants that flexiramp cost function is less than the regulation cost, and leads to a monotonically increasing stepwise or piecewise function as shown in Fig. 2 [2].

The stepwise curve, setting procurement prices for each block of the flexiramp, is expressed as:

$C_{t}^{(\text {frus.frds) }}\left(F R U S_{t}, F R D S_{t}\right)=\sum_{f=1}^{F} c_{f}^{(\text {frus.frds) }} \cdot \chi_{f, t}$

$0 \leqslant \chi_{f, t}^{(u p, d n)} \leqslant \chi_{f}^{\max ,(u p, d n)}$

$\sum_{f=1}^{F} \chi_{f, t}^{(u p, d n)}=F R U S_{t}, F R D S_{t}$

\section{Day-ahead stochastic scheduling}

\subsection{Demand, wind and net load probability density function generation}

The term net load refers to the actual demand minus generation from wind resources. In this paper, the day-ahead load forecast error, follows a normal distribution with zero mean and a standard deviation of $2 \%$ of the peak load. Wind power forecast is derived from the ARMA model, based on wind measurements in the province of Ontario, Canada [15]. Hourly wind generation forecasts are fitted into probability density function (PDFs) with generalised extreme value distribution for low level ( $<0.3$ p.u.) of forecasted wind generation, and Beta distribution for the remaining wind power forecast. The sampling rate, set to $0.05 \mathrm{p} . \mathrm{u}$, is chosen based on the Nyquist rate theorem, to ensure minimal loss of data throughout the discretisation process, and maintaining the reliability of the uncertainty modelling for short-term scheduling [16].

Wind power PDF will be reshaped, by introducing a set binary variable $y_{m t}$ and decision variable $p_{c}^{w}$. The latter has the property that wind curtailment occurs at or above $p_{c}^{w}$, and is comprehensively described in [17], which can be expressed as:

$\bar{p}_{m t}^{w}=p_{m t}^{w} \cdot\left(\sum_{c>m} y_{c t}\right)+y_{m t} \cdot\left(\sum_{c \geqslant m} p_{c t}^{w}\right)$

$\sum_{m} y_{m t}=1$

The PDF of net load is then calculated by convoluting respective discretised PDFs of the demand and the post-curtailment wind power, expressed as:

$d_{s t}^{n e t}=d_{h t}-p_{m t}^{w}, \quad \forall s=M-m+h$

$p_{s, t}^{n e t}=\sum_{\forall h, m: s=M-m+l}^{S} \bar{p}_{m, t}^{w} p_{h, t}^{d}$

Other wind forecasting techniques can be also incorporated on the developed approach without difficulty.

\subsection{Chance-constrained programming to underpin flexibility}

Various stochastic optimization approaches were proposed to deal with wind power uncertainty in generation scheduling: analytical approach [18], fully stochastic (FS) [19], chance-constrained programming (CCP) [20-22], and artificial intelligence method [23]. CCP is proposed herein to model the net load uncertainty and fulfill the flexiramp requirements with a predefined confidence level. In this paper, CCP utilizes a discrete PDF, which is markedly improved from our previous work [24], to better model the day-ahead flexiramp surplus procurement. Three binary variable sets $(\alpha, \beta$ and $\gamma)$ are defined with the properties:

$\min \left\{\delta=\sum_{t=1}^{T} \sum_{s=1}^{S} \gamma_{s, t} \cdot d_{s, t}^{n e t}\right\}$

$\sum_{s=1}^{S} \gamma_{s, t}=1$

$\sum_{s=1}^{S} \gamma_{s, t} \cdot d_{s, t}^{n e t} \geqslant \bar{d}_{t}^{n e t}$

$\beta_{s, t} \geqslant \gamma_{s, t}$

Table 1

Price calculation of flexiramp demand curve.

\begin{tabular}{lllll}
\hline $\begin{array}{l}\text { Flexible ramp } \\
\text { range (MW) }\end{array}$ & $\begin{array}{l}\text { Surplus range } \\
(\mathrm{MW})\end{array}$ & Prob. & $\begin{array}{l}\text { Penalty } \\
(\$ / \mathrm{MWh})\end{array}$ & Price $(\$ / \mathrm{MWh})$ \\
\hline $\begin{array}{l}\text { Flexible ramp up } \\
0-100\end{array}$ & $300-400$ & 0.450 & 1000 & $0.450 * 1000=\$ 450$ \\
$100-200$ & $200-300$ & 0.046 & 1000 & $0.046 * 1000=\$ 46$ \\
$200-300$ & $100-200$ & 0.005 & 1000 & $0.005 * 1000=\$ 5$ \\
$300-400$ & $0-100$ & 0.001 & 1000 & $0.001 * 1000=\$ 1$ \\
Flexible ramp down & & & & \\
$300-400$ & $0-100$ & 0.002 & 150 & $0.002 * 150=\$ 0.3$ \\
$200-300$ & $100-200$ & 0.006 & 150 & $0.006 * 150=\$ 0.9$ \\
$100-200$ & $200-300$ & 0.050 & 150 & $0.050 * 150=\$ 7.5$ \\
$0-100$ & $300-400$ & 0.440 & 150 & $0.440 * 150=\$ 66$ \\
\hline
\end{tabular}




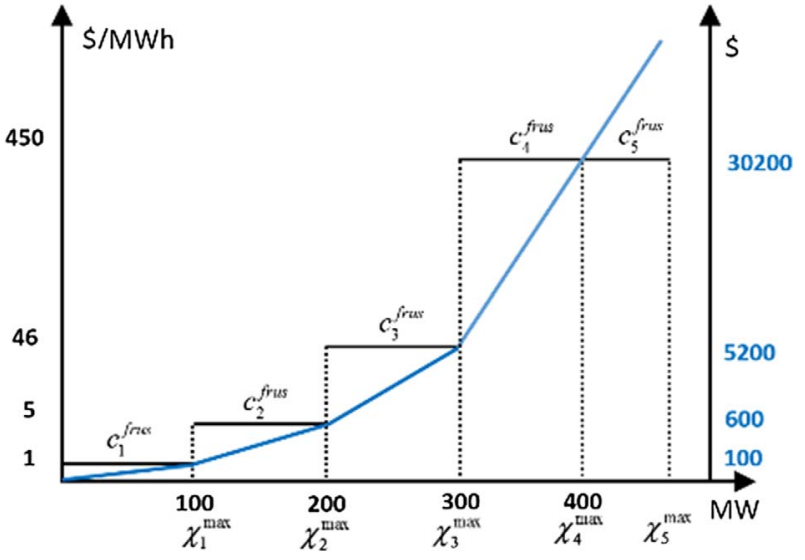

Fig. 2. Step-wise and piece-wise upward flexiramp surplus price curve.

$\beta_{s-1, t} \leqslant 1-\gamma_{s, t}$

$\beta_{s, t} \geqslant \beta_{s-1, t}$

$\alpha_{s, t}=1-\beta_{s, t}$

Eq. (18) is included in the objective function as an auxiliary function $\delta$. The binary $\gamma$ acts as a reference point to the expected net load at time $t$, as shown in Fig. 3a. $\alpha$ and $\beta$ are binary variables, representing down and up flexiramp regions respectively, by setting the value to 1 , as depicted in Fig. 3b, defined by (19)-(24). The chance constraints, with wind curtailment effect, for the day-ahead flexiramp procurement can be represented as:

$\operatorname{Pr}\left(\sum_{i, j=1}^{I, J}\left[F R U_{i, t}+F R U_{j, t}\right] \geqslant d_{s, t+1}^{n e t}-\bar{d}_{t}^{n e t}\right) \geqslant 1-\varepsilon^{u p} \cdot \sum_{s}^{S} p r_{s, t+1} \cdot \beta_{s, t}$

$\operatorname{Pr}\left(\sum_{i, j=1}^{I, J}\left[F R D_{i, t}+F R D_{j, t}\right] \geqslant \bar{d}_{t}^{n e t}-d_{s, t+1}^{n e t}\right) \geqslant 1-\varepsilon^{d n} \cdot \sum_{s}^{S} p r_{s, t+1} \cdot \alpha_{s, t}$

Eqs. (25) and (26) require that the up/down flexiramp appropriations (cycliramp plus flexiramp surplus and reserve) are allocated to fulfill the flexiramp requirement at time $t$, up to a preset up/down confidence level. The $\varepsilon^{(u p, d n)}$ is multiplied with the summation of up/ down net load ramp (NLR) realizations respectively. For instance, setting $\varepsilon^{u p}$ to 0.4 , means $60 \%$ of upward NLR realizations and all the downward $(\alpha)$ NLR realizations are fulfilled.

\subsection{Projected disjunctive reformulation (PDR) approach}

An MILP model, based on a projected disjunctive reformulation (PDR) approach [24], is adopted to linearize the chance constraints (25) and (26). PDR, basically, aims to reduce the problem size by a variable elimination procedure that is equivalent to projection. It also expedites the computation to achieve faster simulation over other CCP MILP formulations, as demonstrated in [24]. First, for scenario dimension, $n \subset\{1, \ldots, N\}$ continuous variable, $G \in \mathfrak{R}^{n}$, let

$v(g)=\left\{s \in\{1, \ldots, S\}: g<d_{n s, t+1}^{\text {net }}\right\}$

$\widetilde{G}_{n}=\left\{g \in \prod_{n \in n}\left\{d_{n s(t+1)}^{n e t}\right\}_{s=1}^{S}:\left|v_{n}(g)\right| \leqslant \varepsilon\right\}$

where $\Pi$ denotes the Cartesian product, and $v_{N}(G)$ is the set of scenarios for which $g$ violates constraint $g \geqslant d_{n s, t+1}^{\text {net }}$.

The PDR approach is applied to the chance constraints (25) and (26), as follows:

$\sum_{i, j=1}^{I, J}\left[F R U_{i, t}+F R U_{j, t}\right]+\bar{d}_{s, t}^{n e t} \geqslant \sum_{h \in \widetilde{G}_{N}} y_{h, t}^{n,(u p, d n)} \cdot g_{h, t}$

$$
\begin{aligned}
& \sum_{i, j=1}^{I, J}\left[F R D_{i, t}+F R D_{j, t}\right]-\bar{d}_{s, t}^{n e t} \geqslant \sum_{h \in \widetilde{G}_{N}} y_{h, t}^{n,(u p, d n)} \cdot g_{h, t} \\
& \sum_{h \in \widetilde{G}_{N}} y_{h, t}^{n,(u p, d n)}=1 \\
& \sum_{s=1}^{S} z_{s, t}^{(u p, d n)} \cdot p r_{s, t} \leqslant \varepsilon^{(u p, d n)} \cdot \sum_{s}^{S} p r_{s, t+1} \cdot\left(\alpha_{s, t}, \beta_{s, t}\right) \\
& z_{s, t}^{(u p, d n)} \geqslant \sum_{h: s \in v_{n}(g)} y_{h, t}^{n,(u p, d n)}
\end{aligned}
$$

The MILP formulation in (29)-(33) still contains a nonlinearity, namely the product of binary variable $z_{s, t}^{(u p, d n)}$, with the continuous variable $p r_{s t}$. Reformulation can be performed by defining the continuous variable, $\omega_{s, t}^{(u p, d n)}$, in which $\omega_{s, t}^{(u p, d n)}=z_{s, t}^{(u p, d n)} \cdot p r_{s, t}$, subject to the following constraints:

$0 \leqslant \omega_{s, t}^{(u p, d n)} \leqslant z_{s, t}^{(u p, d n)}$

$0 \leqslant p r_{s, t}-\omega_{s, t}^{(u p, d n)} \leqslant 1-z_{s, t}^{(u p, d n)}$

\subsection{Day-ahead scheduling problem formulation}

The proposed multi-timescale scheduling consists of CCP DASO and deterministic RTDO model. DASO incorporates load demand and wind uncertainty, with the objective function to minimize total operation cost (generation, start-up, shut-down, cycliramp), flexiramp surplus procurement cost, and ES charge/discharge cost.

$$
\begin{aligned}
& \min \left\{\sum_{t=1}^{T} \sum_{i=1}^{I}\left[C_{i, t}^{g}\left(p_{i, t}\right)+C_{i, t}^{s u}+C_{i, t}^{s d}+C_{i, t}^{r}\left(C R U_{i, t}, C R D_{i, t}\right)\right]\right. \\
& \left.+\sum_{t=1}^{T}\left[C_{t}^{\text {frus }}\left(F R U S_{t}\right)+C_{t}^{\text {frds }}\left(F R D S_{t}\right)\right]+\sum_{t=1}^{T} \sum_{j=1}^{J}\left[C_{j, t}^{e s}\left(q_{j, t}^{c}, q_{j, t}^{d}\right)\right]+\delta\right\}
\end{aligned}
$$

s.t.

$C_{i, t}^{g}\left(p_{i, t}\right)=p_{i, t} \cdot h r_{i}^{g} \cdot \pi_{i}^{g}$

$C_{i, t}^{s u} \geqslant S U_{n, i} \cdot\left(o_{i, t}-\sum_{k=1}^{n} o_{i, t-k}\right), C_{i, t}^{s u} \geqslant 0$

$C_{i, t}^{s d} \geqslant S D_{i} \cdot\left(o_{i, t-1}-o_{i, t}\right), C_{i, t}^{s d} \geqslant 0$

$C_{j, t}^{e s}\left(q_{j, t}^{c}, q_{j, t}^{d}\right)=\pi_{j}^{c} \cdot q_{j, t}^{c}+\pi_{j}^{d} \cdot q_{j, t}^{d}$

$\sum_{i}^{I} p_{i, t}^{g}+p_{t}^{w}+\sum_{j=1}^{J} q_{j, t}^{d}-\sum_{j=1}^{J} q_{j, t}^{c}=\sum_{b=1}^{B} d_{b, t}$

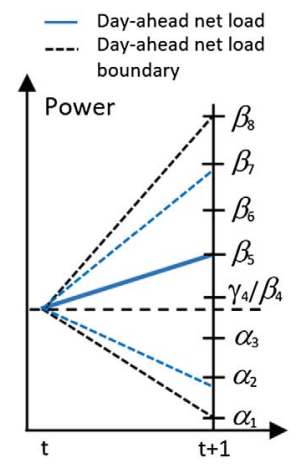

(a) Net load ramp

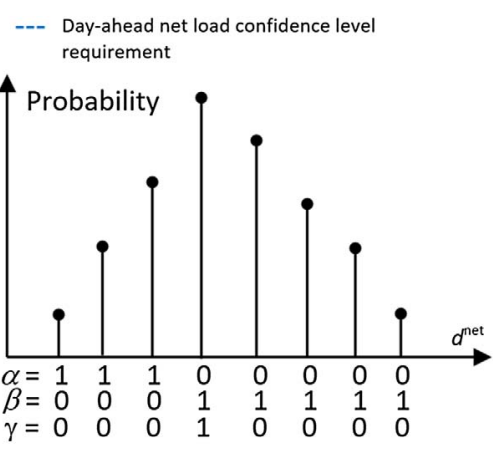

(b) Discrete probability density function
Fig. 3. Proposed chance constraint flexiramp procurement model. 
$-P L^{\max } \leqslant S F \times\left[K_{G} \times P^{G}-K_{D} \times P^{D}\right] \leqslant P L^{\max }$

$\sum_{i=1}^{I} p_{i}^{\max } \cdot o_{i, t} \geqslant \sum_{b=1}^{B} d_{b, t}+s r_{t}$

$p_{i, t+1}-p_{i, t} \leqslant r_{i, t}^{u p} \cdot o_{i, t}+p_{i}^{\min } \cdot\left(o_{i, t+1}-o_{i, t}\right)$

$p_{i, t}-p_{i, t+1} \leqslant r_{i, t}^{d n} \cdot o_{i, t+1}+p_{i}^{\min } \cdot\left(o_{i, t}-o_{i, t+1}\right)$

$p_{i}^{\min } \cdot o_{i, t} \leqslant p_{i, t} \leqslant p_{i}^{\max } \cdot o_{i, t}$

$-o_{i, t-1}+o_{i, t}-o_{i, k} \leqslant 0, \forall 1 \leqslant k-(t-1) \leqslant T_{i}^{o n}$

$o_{i, t-1}-o_{i, t}+o_{i, k} \leqslant 1, \forall 1 \leqslant k-(t-1) \leqslant T_{i}^{o f f}$

$q_{j, t}^{\min , c} \cdot\left(1-x_{j, t}\right) \leqslant q_{j, t}^{c} \leqslant q_{j, t}^{\max , c} \cdot\left(1-x_{j, t}\right)$

$q_{j, t}^{\min , d} \cdot x_{j, t} \leqslant q_{j, t}^{d} \leqslant q_{j, t}^{\max , d} \cdot x_{j, t}$

$e_{j, t}=e_{j, t-1}+\left[\eta_{j}^{c} \cdot q_{j, t}^{c}-\left(1 / \eta_{j}^{d}\right) \cdot q_{j, t}^{d}\right] \cdot\left(1 / e_{j}^{\max }\right)$

$e_{j}^{\min } / e_{j}^{\max } \leqslant e_{j, t} \leqslant 1$

$e_{j, 0}=e_{j, T}=E_{j}^{0}$

Thermal unit production cost, start-up cost, shut-down cost, and ES operation cost are detailed in (37)-(40). Since the cycliramp cost and flexiramp surplus cost functions are inversely proportional, the inclusion of both of them in the objective function, (36), is crucial to ensure reducing the wear and tear of conventional thermal generation, as well as maintaining minimal flexiramp surplus procurement. System-wide constraints entail power balance constraints (41) and transmission line limits (42). System spinning reserve requirement is included in (43). Eqs. (44) and (45) are generation ramp up and ramp down limits. Eq. (46) is the generation output limits. Eqs. (47) and (48) are generation minimum on/off time limits. Other generation constraints are the initial generation on/off limits [25]. ES constraints include charge and discharge power limits, state of charge (SOC) dynamics, SOC limits, initial/final SOC limits, which are defined in (49)-(52), respectively. Constraint (53) presumes that the ES follows a daily cycle, as the initial time $(t=0)$ and the last hour $(t=T)$ should be the same.

The nonlinear production cost of thermal units (37) is approximated by a piecewise function, whereas start-up cost follows a stepwise function [25]. Dynamic stepwise ramp rates are also included [26]. Solving the above model (1)-(53), gives a complete stochastic dayahead scheduling problem.

\section{Real-time deterministic scheduling}

RTDO aims to adjust the generation scheduled in the day-ahead according to the newly predicted load demand and wind generation. RTDO is a 3-h ahead online rolling scheduling that runs iteratively every hour. The proposed RTDO objective function, (54), optimizes the deviation of each day-ahead parameter to meet real-time net load changes.

$$
\begin{aligned}
& \min \left\{\sum _ { m = 1 } ^ { M } \sum _ { i = 1 } ^ { I } \left[\tau \cdot C_{i, m}^{g}\left(p_{i, m}\right)+\tau \cdot C_{i, m}^{r}\left(C R U_{i, m}, C R D_{i, m}\right)\right.\right. \\
& +\sum_{m=1}^{M}\left[\tau \cdot C_{m}^{f r u s}\left(F R U S_{m}\right)+\tau \cdot C_{m}^{f r d s}\left(F R D S_{m}\right)\right] \\
& \left.+\sum_{m=1}^{M} \sum_{j=1}^{J}\left[\tau \cdot C_{j, m}^{e s}\left(q_{j, m}^{c}, q_{j, m}^{d}\right)\right]\right\}
\end{aligned}
$$

s.t.

$\Delta_{i, m}^{p g} \geqslant \max \left(p_{i}^{\min } \cdot o_{i, m}-p_{i, m}^{s c h}, p_{i, t}^{D A}-p_{i, m}^{s c h}-\Delta_{i}^{\max , p g}\right)$

$\Delta_{i, m}^{p g} \leqslant \min \left(p_{i}^{\max } \cdot o_{i, m}-p_{i, m}^{s c h}, p_{i, t}^{D A}-p_{i, m}^{s c h}+\Delta_{i}^{\max , p g}\right)$

$-\Delta^{\text {max frus }} \leqslant F R U S_{m}^{\text {sch }}+\Delta_{m}^{\text {frus }}-F R U S_{t}^{D A} \leqslant \Delta^{\text {max frus }}$

$-\Delta^{\text {max } f r d s} \leqslant F R D S_{m}^{s c h}+\Delta_{m}^{f r d s}-F R D S_{t}^{D A} \leqslant \Delta^{\text {max } f r d s}$

$\Delta_{j, m}^{e} \geqslant \max \left(e_{j}^{\min }-e_{j, m}^{s c h}, e_{j, t}^{D A}-e_{j, m}^{s c h}-\Delta_{j}^{\max , e}\right)$

$\Delta_{j, m}^{e} \leqslant \min \left(e_{j}^{\max }-e_{j, m}^{s c h}, e_{j, t}^{D A}-e_{j, m}^{s c h}+\Delta_{j}^{\max , e}\right)$

Notably, the flexiramp, system-wide, generation and storage constraints are identical to the DASO (1)(13) and (37)(53), only with the add-on of decision variable $\Delta_{m}$ to every scheduled parameter; i.e., thermal generation, flexiramp surplus, and storage charge/discharge rates. Cost functions are multiplied by intra-hour rate of $\tau=0.25$ in the 15-min time-scale RTSO. Additional constraints (55)-(60) state that the deviation of the respective scheduled parameters between RTSO and DASO should be within a limited range. The day-ahead preset values, viz. real-time flexiramp requirements, $\phi^{u p(d n)}$ are determined by considering normal distribution net load forecast mean error, $\mu^{u p(d n)}$, and standard deviation, $\sigma^{u p(d n)}$. The $2 \sigma$ rule, used to cover approximately $95 \%$ net load variability and uncertainty [27], is expressed as:

$\phi^{u p}=2 \sigma_{m}^{u p}+\mu_{m}^{u p}$

$\phi^{d n}=2 \sigma_{m}^{d n}+\mu_{m}^{d n}$

\section{Numerical results}

Two case studies comprising a 6-bus system and IEEE 118-bus system are presented to demonstrate the effectiveness of the multitimescale model. The resulting MILP problem is solved by $\mathrm{C}++$ /CPLEX

\begin{tabular}{|c|c|c|c|c|c|c|c|c|c|c|c|}
\hline Case & Bus & Con. lvl. (\%) & Prod. cost (\$) & FRS cost (\$) & CR cost (\$) & ES cost $(\$)$ & Total cost $(\$)$ & FRUR (MW) & FRDR (MW) & Wind crtl. (\%) & Comp. time (s) \\
\hline 1-DA & 6 & - & 101,887 & - & 140 & - & 102,027 & - & - & - & 1 \\
\hline \multirow[t]{2}{*}{ 2-DA } & 6 & 75 & 76,628 & 304 & 53 & - & 76,985 & 1221 & 608 & 11.46 & 48 \\
\hline & & 95 & 77,214 & 313 & 46 & - & 77,573 & 1357 & 865 & 13.52 & 81 \\
\hline \multirow[t]{2}{*}{ 3.1-DA } & 6 & 75 & 77,893 & 318 & 271 & - & 78,482 & 1465 & 598 & 11.40 & 74 \\
\hline & & 95 & 79,047 & 320 & 260 & - & 79,627 & 1502 & 706 & 13.66 & 79 \\
\hline \multirow[t]{2}{*}{ 3.2-DA } & 6 & 75 & 73,950 & 277 & 64 & 16 & 74,307 & 2869 & 1945 & 0.00 & 26 \\
\hline & & 95 & 73,950 & 277 & 64 & 16 & 74,307 & 2869 & 1945 & 0.00 & 31 \\
\hline 4.1-DA & 118 & - & 852,170 & - & 772 & - & 852,942 & - & - & - & 31 \\
\hline \multirow[t]{2}{*}{ 4.2-DA } & 118 & 75 & 662,892 & 4634 & 616 & - & 668,142 & 42,576 & 20,498 & 1.32 & 281 \\
\hline & & 95 & 666,548 & 4741 & 581 & - & 671,870 & 41,350 & 21,429 & 3.01 & 751 \\
\hline \multirow[t]{2}{*}{ 4.3-DA } & 118 & 75 & 656,090 & 4688 & 556 & 109 & 661,531 & 54,210 & 22,276 & 0.78 & 198 \\
\hline & & 95 & 658,297 & 4706 & 538 & 107 & 663,650 & 48,053 & 25,291 & 2.14 & 366 \\
\hline \multirow[t]{2}{*}{ 4.4-DA } & 118 & 75 & 654,136 & 4800 & 499 & 212 & 659,647 & 68,065 & 40,102 & 0.00 & 184 \\
\hline & & 95 & 654,226 & 4899 & 483 & 167 & 659,775 & 65,337 & 41,452 & 0.00 & 197 \\
\hline
\end{tabular}

Table 2

Expected day-ahead system economics. 
12.6, with $3.20 \mathrm{GHz}$ Intel Core i5 and $10 \mathrm{~GB}$ RAM memory.

\subsection{Six-bus system}

The 6-bus system data consists of 3 thermal units, 1 wind farm, 7 lines and 3 loads, which are given in [11]. The cycliramp penalty cost is set to $\$ 0.25 / \mathrm{MWh}$ for normal ramp and $\$ 0.50 / \mathrm{MWh}$ if the ramp exceeds the elastic limit of the generator (extreme ramp) [5]. A $150 \mathrm{MW}$ wind farm with $20 \%$ wind penetration level and a 150MWh ES is included at bus 5 and 4, respectively. The daily wind profile is taken from EirGrid system data on March 24th 2016 (15-min intervals) [28]. The predicted wind output is normalized to obtain the wind curve. The charge/discharge cost of the ES unit is assumed to be \$0.5/MWh and $\$ 0.1 / \mathrm{MWh}$, respectively. The initial energy level of the ES unit is set to $50 \%$ of its capacity (i.e., $75 \mathrm{MWh}$ ). The flexiramp surplus procurement preset value $\phi$, estimated based on (61) and (62), is fixed to $14 \mathrm{MW}$ (95\% of real-time forecast error). Three test cases are considered: (1) Base case with only thermal units. (2) Impact of flexiramp surplus procurement on thermal unit scheduling. (3) Impact of flexiramp, cycliramp and ES on multi-timescale scheduling.

Case 1. The base case is run without considering flexiramp surplus cost, cycliramp cost, ES and wind generation, to provide a comparison platform for other test cases. As cycliramp cost is not considered in the objective function, it is calculated on a posteriori basis. As shown in Table 2, case 1, day-ahead operation cost is $\$ 102,027$ which includes $\$ 101,887$ production cost, and $\$ 140$ cycliramp cost. The real-time production cost is $\$ 100,517$ and the cycliramp cost is $\$ 60$, as shown in Table 3. These minor cost variations are mainly due to load forecast error.

Case 2. The proposed HSD-MTS is simulated with $20 \%$ wind penetration and various levels of flexiramp procurement. The effect of flexiramp procurement to thermal generation scheduling is validated by tuning the CCP confidence level. Both the PDR and Big-M exactly linearize the CCP without approximation, so that the CCP can be theoretically solved to optimality. Therefore, both approaches converge to the same scheduling results. The CCP is adjusted to $0.1 \%$ optimality gap for the 6-bus system.

Simulation results, in Tables 2 and 3, clearly show production cost experiencing a substantial reduction as wind power is incorporated. Nonetheless, for DASO, as the confidence level increases, signifying an increase in the demand of flexiramp capacity, production cost increases; due to opportunity cost and wind curtailment. As a result, thermal units experience additional ramping, to provide sufficient flexiramp capacity at each time $t$. Wind curtailment also increases, when higher flexibility is enforced, mainly as thermal unit flexiramp capacity is insufficient to meet the higher net load uncertainty. In hours of low system load, wind curtailment occurs when there is insufficient down flexiramp, by increasing the demand in the valley of net demand curve, which leads to a flatter net load curve, thus wind-induced cycliramp cost subsequently decreases.

To preserve the cost effectiveness of the flexiramp product and avoid the over-procurement of flexiramp reserve that leads to higher opportunity cost, the $75 \%$ confidence level, Table 2 , is chosen as the base input for the RTDO, listed in Table 3. This confidence level provides sufficient flexiramp reserve to deal with real-time uncertainties. Actual wind power is lower than the day-ahead forecasted wind; causing Case 2 RTDO production cost to increase, as compared with DASO, even with lower wind curtailment. Flexiramp surplus procurement in the RTDO is comparable to the day-ahead, since the preset DASO flexiramp surplus requirement is set to cover the $95 \%$ real-time forecast error uncertainty.

Case 3. The effects of extreme generating unit cycliramp cost (case 3.1), and ES implementation (case 3.2), are discussed in this case. In case 3.1, an excessive cycling ramp penalty factor is applied by setting it to $\$ 25 / \mathrm{MWh}$ and $\$ 50 / \mathrm{MWh}$ for normal and extreme ramp respectively.
Fig. 4 shows DASO's Gen 1 power trajectory and respective cycliramp amount with low/high cycliramp penalty factor, and with/ without ES. In case 3.1, high penalty demonstrates a flatter power trajectory, as well as a rise in thermal generation cost. This is basically due to the utilization of Gen 2, to level out the output of Gen 1. In case 3.2, the addition of the ES further reduces production cost, because of the ES capability to store excess wind energy and shift them to another period. Additionally, wind curtailment is completely eliminated with the application of the ES. Furthermore, total flexiramp capacity required to fulfill the chance constraints is increased, thus reducing the opportunity cost. ES also supplies additional flexiramp capacity that proportionally increases the flexiramp reserve, which will ensure a sufficient and efficient response to the net load deviation between the two timescales. This causes the chance constraints again to become redundant, as the results of $75 \%$ and $95 \%$ confidence levels are equal.

\subsection{8-Bus system}

The IEEE 118-bus system has 54 thermal units, 186 lines, and 91 loads. The parameters of generators, transmission network and load profiles are obtained from [29]. The peak load of $3733 \mathrm{MW}$ occurs at hour 15. Four $550 \mathrm{MW}$ wind turbines are installed at bus 15, 24, 54 and 96 , which contribute to $21.8 \%$ wind penetration. The wind curve pattern, cycliramp penalty cost, ES initial energy level, and charge/discharge cost are the same as the 6-bus system. An optimality gap of $1.5 \%$ and a flexiramp surplus preset value $\phi$ of 206 MW are fixed for the 118bus system.

Case 4. The base case of 118-bus system (case 4.1), with a daily operating cost of $\$ 852,170$, and cycliramp cost of $\$ 772$. The 118-bus system with $21.8 \%$ wind penetration is simulated in case 4.2. Furthermore, the impact of the ES sizing on the multi-timescale scheduling is investigated for the 118-bus system. Four 100MWh ES (case 4.3), and four 500MWh ES (case 4.4) are installed at busses with wind turbine, and the corresponding results are listed in Table 2. Wind curtailment is eliminated with the increase in ES size, due to the utilization of the ES to store wind energy. Production cost is reduced with the increase in wind utilization (Case 4.4). Cycliramp cost reduces with the implementation of ES in Case 4.3, as net load is smoothed by the ES. The larger capacity of the ES, in Case 4.4, further reduces the cycliramp cost, and levels out the net load. Flexiramp reserve has expectedly increased as ES provides large amount of it.

\subsection{Computational requirements}

The computational performance of the proposed HSD-MTS framework is investigated. Tables 2 and 3 depicts the computation time, in case of a sequential processing, of the PDR approach. PDR managed to solve the DASO problem, in case 4.2 , in just $751 \mathrm{~s}$, with $95 \%$ confidence level. In other words, the proposed CCP-based DASO model is capable of providing optimal solutions at fast execution times, even in large scale power systems. This is particularly because of the efficient MILP

Table 3

Expected real-time system economics.

\begin{tabular}{llllllll}
\hline Case & $\begin{array}{l}\text { Prod cost } \\
(\$)\end{array}$ & $\begin{array}{l}\text { FRS } \\
\text { cost } \\
(\$)\end{array}$ & $\begin{array}{l}\text { CR } \\
\text { cost } \\
(\$)\end{array}$ & $\begin{array}{l}\text { ES } \\
\text { cost } \\
(\$)\end{array}$ & $\begin{array}{l}\text { Total cost } \\
(\$)\end{array}$ & $\begin{array}{l}\text { Wind } \\
\text { crtl. }(\%)\end{array}$ & $\begin{array}{l}\text { Total } \\
\text { comp. } \\
\text { time (s) }\end{array}$ \\
\hline 1-RT & 100,517 & - & 60 & - & 100,577 & - & 1.88 \\
2-RT & 81,502 & 231 & 70 & - & 81,803 & 7.72 & 23.57 \\
3.1-RT & 81,699 & 387 & 483 & - & 82,569 & 7.10 & 2.99 \\
3.2-RT & 80,031 & 227 & 78 & 27 & 80,363 & 0.00 & 26.35 \\
$4.1-R T$ & 856,426 & - & 461 & - & 856,887 & - & 16.93 \\
$4.2-R T$ & 711,788 & 5561 & 331 & - & 717,680 & 3.71 & 40.63 \\
$4.3-R T$ & 709,060 & 5617 & 253 & 133 & 715,063 & 0.00 & 63.22 \\
$4.4-R T$ & 707,641 & 5693 & 229 & 519 & 714,082 & 0.00 & 63.29 \\
\hline
\end{tabular}




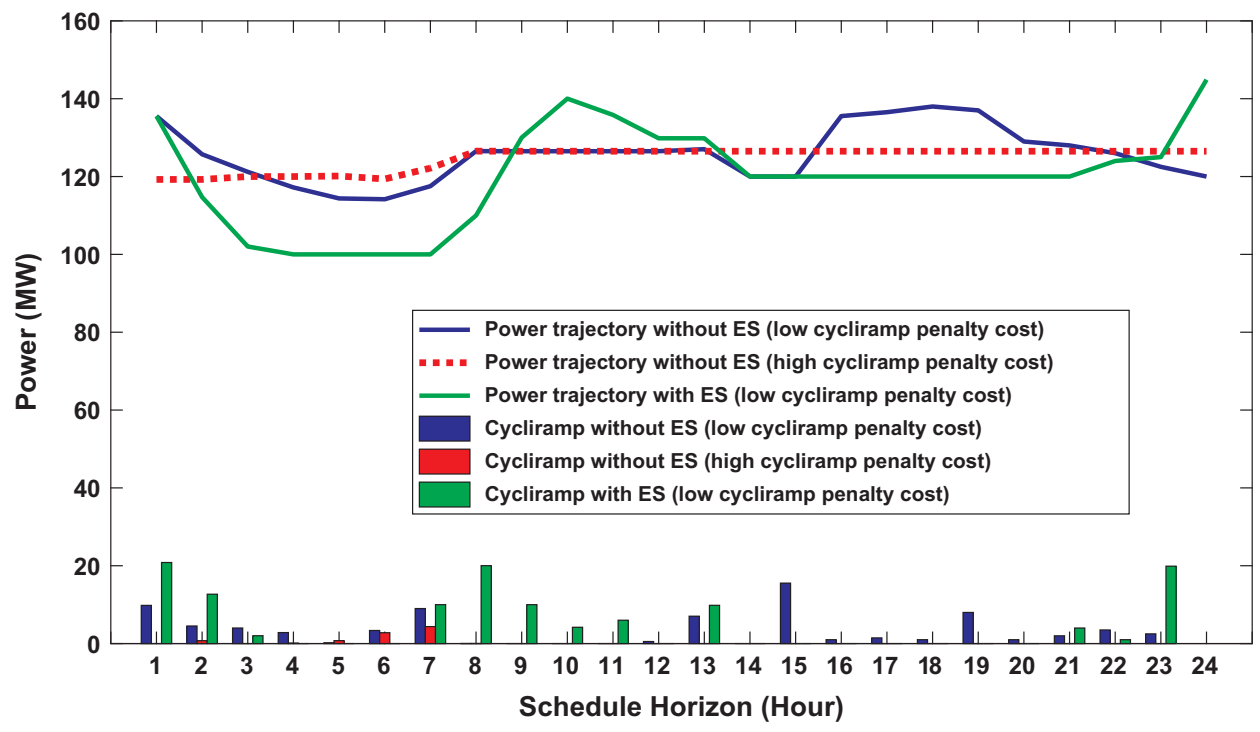

Fig. 4. Power trajectories and cycliramp of Gen 1 in 6-bus system.

structure acquired during the unique factorization process used in the PDR. On the other hand, the online rolling RTDO is solved under an average time of $1 \mathrm{~s}$ and $2.5 \mathrm{~s}$ per iteration, for both 6-bus and 118-bus system, respectively.

One way of improving the computational efficiency of the proposed CCP DASO model is through additional net load discrete realization, to provide higher accuracy solutions. Parallel computing techniques, as well as decomposition techniques, can be effective in this regard to ameliorate the computation time of the PDR [21].

\section{Conclusions}

This paper has proposed a multi-timescale scheduling, with hybrid stochastic day-ahead and deterministic real-time scheduling model, integrating flexiramp surplus and cycliramp costs. The real-time scheduling optimizes only the parameters deviation, based on the day-ahead results to balance out the deviation between predicted and actual net load demand values. Numerical results have shown that high penetration of variable wind generation introduces additional uncertainty in net load demand and a higher requirement for flexiramp and cycliramp. These can be relieved by procuring flexiramp from the generation side and implementing the ES. The elasticity of the proposed model in setting the day-ahead flexiramp surplus procurement, and flexiramp reserve to avoid over procurement, is demonstrated. Increased CCP confidence level may result in higher wind curtailment, higher demand of cycliramp and flexiramp reserve, which would cause an overall higher operation cost. ES capability to shift generation is useful to reduce wind curtailment and flatten the net load, in order to reduce the cycliramp. Minimizing cycliramp costs could level out the hourly generation profiles of thermal generators and reduce the wear and tear.

\section{Acknowledgments}

This work was supported by the Malaysian Ministry of Higher Education (MOHE), and Universiti Malaysia Sarawak (UNIMAS), Malaysia under the Fundamental Research Grant Scheme (FRGS), Grant No. F02/FRGS/1620/2017.

\section{References}

[1] Zhang B, Kezunovic M. Impact on power system flexibility by electric vehicle participation in ramp market. IEEE Trans on Smart Grid. May. 2016;7(3):1285-94.

[2] Scholz M. Business requirements specification - flexible ramping product. http:// www.caiso.com/Documents/BusinessRequirementsSpecification-
FlexibleRampingProduct.pdf.

[3] Xu L, Tretheway D. Flexible Ramping Products - Draft Final Proposal. www.caiso. com/documents/draftfinalproposal-flexiblerampingproduct.pdf.

[4] Navid N, Rosenwald G. Ramp capability product design for MISO markets. https:// www.misoenergy.org/Library/Repository/Communication\%20Material/Key \%20Presentations\%20and\%20Whitepapers/Ramp\%20Capability\%20for\%20Load \%20Following\%20in\%20MISO\%20Markets\%20White\%20Paper.pdf.

[5] Van den Bergh K, Delarue E. Cycling of conventional power plants: technical limits and actual costs. Energy Convers and Management. June. 2015;97:70-7.

[6] Wu H, Shahidehpour M, Khodayar ME. Hourly demand response in day-ahead scheduling considering generating unit ramping cost. IEEE Trans Power Syst. April. 2013;28(3):2446-54.

[7] Costley M, Feizollahi MJ, Ahmed S, Grijalva S. A rolling-horizon unit commitment framework with flexible periodicity. Int J Electr Power Energy Syst 2017;90:280-91.

[8] Marneris I, Biskas P, Bakirtzis A. Stochastic and Deterministic Unit Commitment Considering Uncertainty and Variability Reserves for High Renewable Integration. Energies. 2017;10(1):140.

[9] Wu C, Hug G, Kar S. Risk-limiting economic dispatch for electricity markets with flexible ramping products. IEEE Trans Power Syst. May. 2016;31(3):1990-2003.

[10] Wang B, Hobbs BF. Real-time markets for flexiramp: a stochastic unit commitmentbased analysis. IEEE Trans Power Syst. Mar. 2016;31(2):846-60.

[11] Wu H, Shahidehpour M, Alabdulwahab A, Abusorrah A. Thermal generation flexibility with ramping costs and hourly demand response in stochastic security-constrained scheduling of variable energy sources. IEEE Trans Power Syst. Dec. 2015;30(6):2955-64.

[12] Troy N, Denny E, Malley MO. Base-Load cycling on a system with significant wind penetration. IEEE Trans on Power Syst. May. 2010;25(2):1088-97.

[13] Huang R, Farantatos E, Cokkinides GJ, Meliopoulos AP. Impact of non-dispatchable renewables on generator cycling and control via a hierarchical control scheme. Transmission and Distribution Conference and Exposition (T\&D), 2012 IEEE PESMay. 7-10, 2012. p. 1-8.

[14] Troy N, Flynn D, Milligan M, Malley MO. Unit commitment with dynamic cycling costs. IEEE Trans Power Syst. Nov. 2012;27(4):2196-205.

[15] AWS Truewind. A Report to the Ontario Power Authority (OPA) WIND GENERATION DATA. http://www.powerauthority.on.ca/integrated-power-systemplan/simulated-wind-generation-data.

[16] Lyons RG. Understanding Digital Signal Processing: Pearson. Education 2010.

[17] Restrepo JF, Galiana FD. Assessing the Yearly Impact of Wind Power Through a New Hybrid Deterministic/Stochastic Unit Commitment. IEEE Trans Power Syst. 2011;26(1):401-10.

[18] Kalantari A, Galiana FD. Generalized Sigma approach to unit commitment with uncertain wind power generation. Int J Electr Power Energy Syst 2015;65:367-74.

[19] Osório GJ, Lujano-Rojas JM, Matias JCO, Catalão JPS. A new scenario generationbased method to solve the unit commitment problem with high penetration of renewable energies. Int J Electr Power Energy Syst 2015;64:1063-72.

[20] Wu Z, Zeng P, Zhang XP, Zhou Q. A solution to the chance-constrained two-stage stochastic program for unit commitment with wind energy integration. IEEE Trans Power Syst. Jan. 2016;31:4185-96.

[21] Restrepo JF, Galiana FD. Assessing the yearly impact of wind power through a new hybrid deterministic/stochastic unit commitment. IEEE Trans on Power Syst. Feb. 2011;26(1):401-10.

[22] Hreinsson K, Vrakopoulou M, Andersson G. Stochastic security constrained unit commitment and non-spinning reserve allocation with performance guarantees. Int J Electr Power Energy Syst 2015;72:109-15.

[23] Govardhan M, Roy R. Economic analysis of unit commitment with distributed 
energy resources. Int J Electr Power Energy Syst 2015;71:1-14.

[24] Tan W-S, Shaaban M. A hybrid stochastic/deterministic unit commitment based on projected disjunctive MILP reformulation. IEEE Trans Power Syst. Feb. 2016;31:5200-1.

[25] Carrión M, Arroyo JM. A computationally efficient mixed-integer linear formulation for the thermal unit commitment problem. IEEE Trans Power Syst. Aug. 2006;21(3):1371-8.

[26] Li T, Shahidehpour M. Dynamic ramping in unit commitment. IEEE Trans Power
Syst. Aug. 2007;22(3):1379-81.

[27] Marneris IG, Biskas PN, Bakirtzis EA. An integrated scheduling approach to underpin flexibility in European power systems. IEEE Trans on Sustain Energy. Apr. 2016;7(2):647-57.

[28] Eirgrid Group. Eirgrid system information. http://www.eirgridgroup.com/how-thegrid-works/system-information/.

[29] Yong F, Shahidehpour M, Zuyi L. Security-constrained unit commitment with AC constraints. IEEE Trans on Power Syst. May. 2005;20(2):1001-13. 